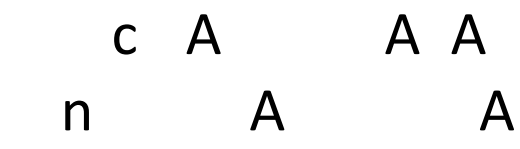

A

J) Glohal Journal of $\begin{array}{lllllllll}W & n & A & ; A & A & ; A & {[A} & A\end{array}$

http://sproc.org/ojs/index.php/gjcs

\title{
E-Tendering: Modeling of a multi-agents system integrating the concepts of ontology and big data
}

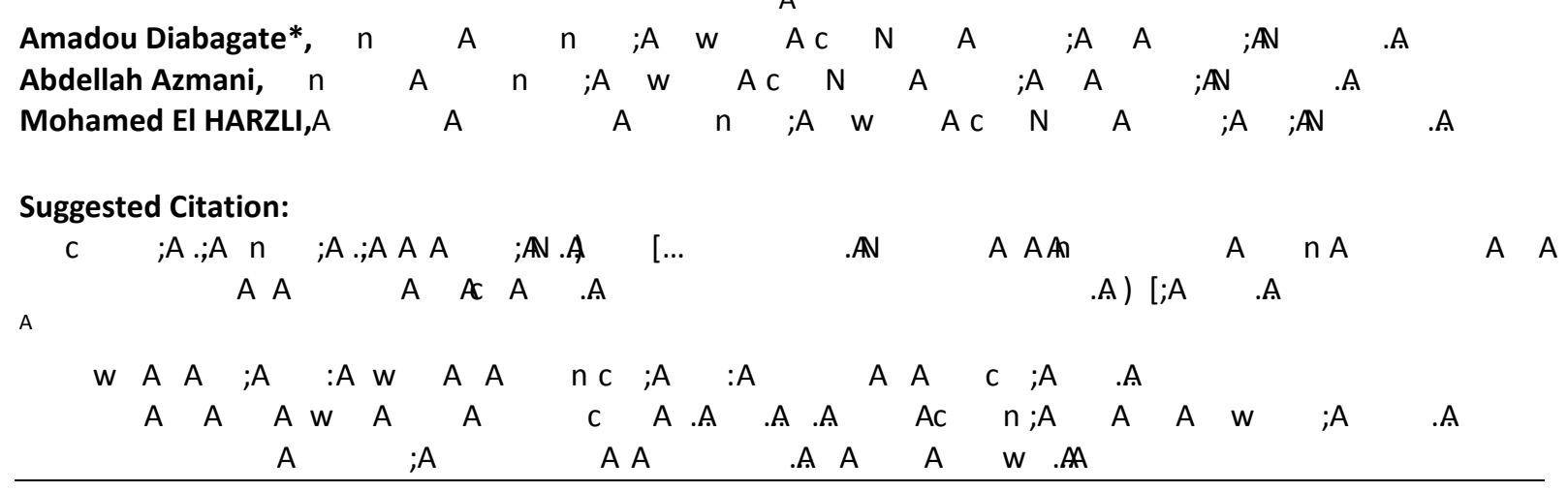

Abstract

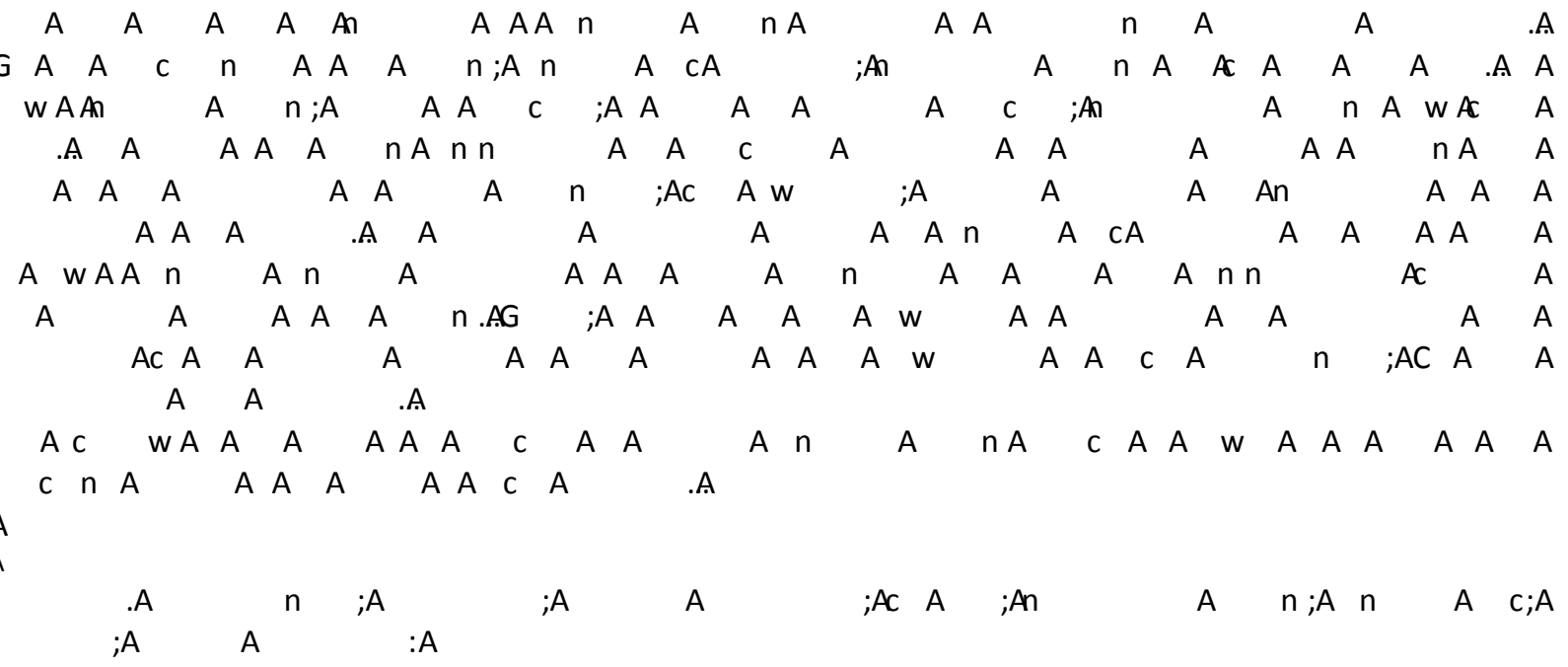

AG A

$; \mathbb{A} \quad$ A
A

A 


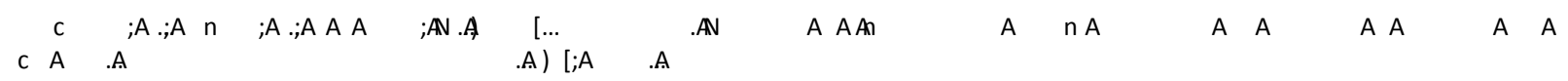

\section{Introduction}

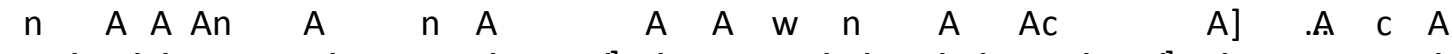

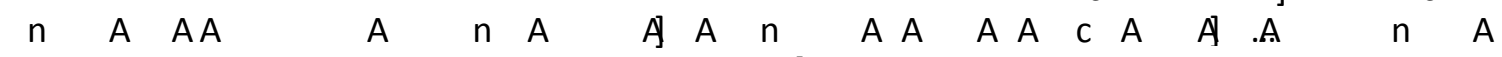

$$
\begin{aligned}
& \text { A } A \text { A } A \cap \text { A A A A A A ACA A A A A A } \\
& \text { An } A A r \text { A A } ; A \text { w } A \text { A A A } A \text { A } A \text { A } A \text { w } A
\end{aligned}
$$

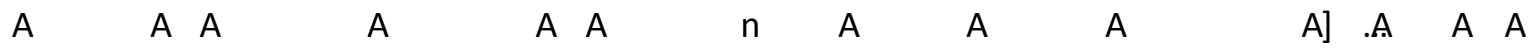

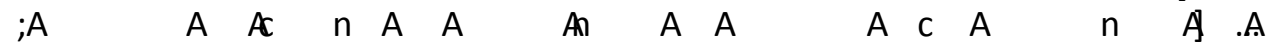

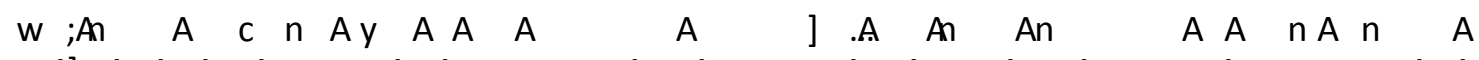

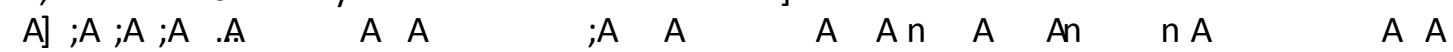

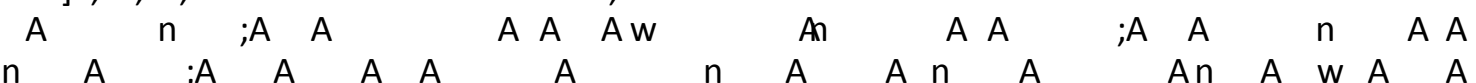

$$
\begin{aligned}
& \begin{array}{lllllllllll}
n & n & n & A & A & n & A & . A
\end{array}
\end{aligned}
$$

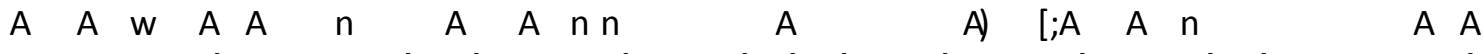

$$
\begin{aligned}
& \begin{array}{lllllllllllllllll} 
& A & n & A & A & A c & n & ; A & A & A & A & A & W A & ; A & A & & \text { C }
\end{array} \\
& A \begin{array}{lllllll}
A & C & \cap & A & A & ; A & \text {.A }
\end{array}
\end{aligned}
$$

A

2. System's general overview

A A Y A A A n $\begin{array}{lllllllll}A & A & C & A & n & ; A & A & A & A\end{array}$ A $A A n \quad A \quad n A$ AAw A $A \quad W \quad A \quad A \quad A C \quad W \quad A$

- $\mathrm{N}$ A A A $\quad A$

- $\quad$ w A A A $A$

- C a A A A A $\quad$ A A

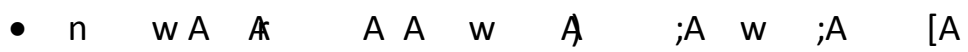

$\begin{array}{llllllllllll} & n & A & A & A & A & c & A & A & c & A & n\end{array}$

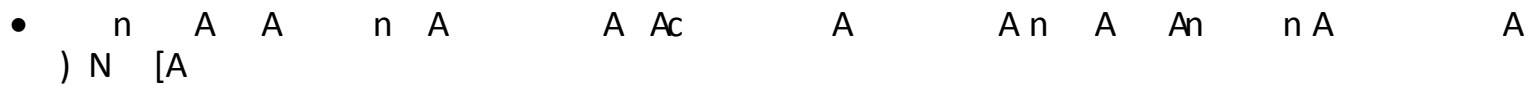
A
A AA A A
A A A $\mathrm{A} . \mathrm{AA}$
A A
A .A

- w a $: A$

- $A$ a A a :A

- $A n A$ A Ay A :A 


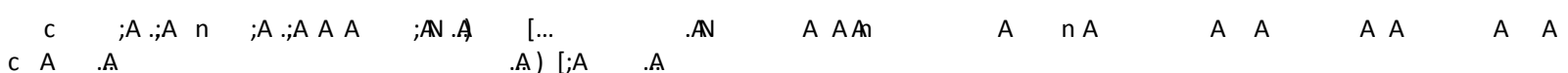

- $\quad \mathrm{A} \quad \mathrm{A} \quad \mathrm{n} \cdot \mathrm{A}$

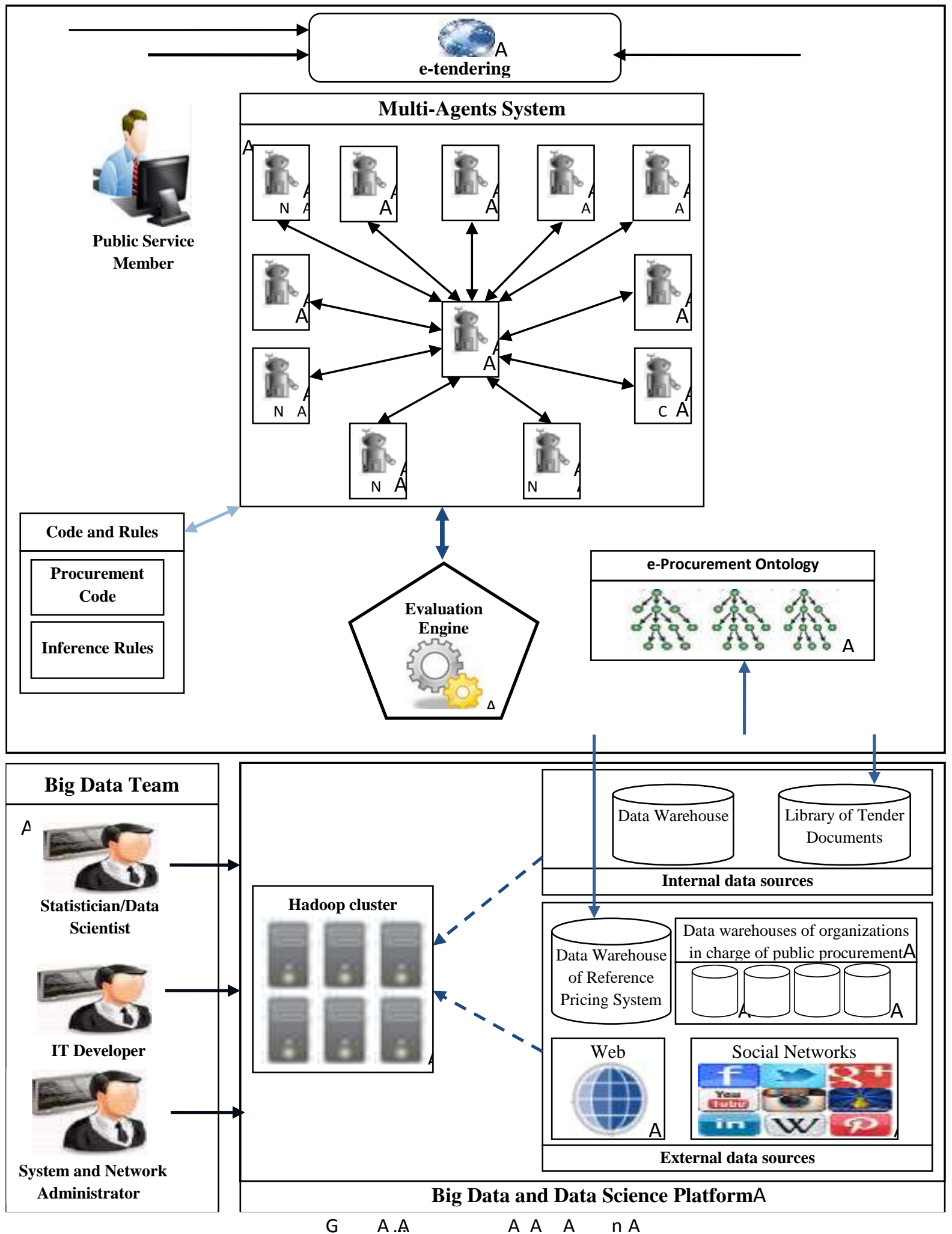

A

$G \cap A \quad A$

A A A A A

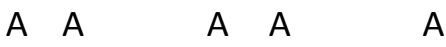

${ }^{A}$

A A

A A A A

$A \quad A n n$

$\begin{array}{ccccc}A & n ; A & A & A & A \\ A & A & & A & A\end{array}$

A $C A$

A A

C A $A$

A

C A A A A

A A

$\mathrm{A} A \quad ; \mathrm{A} A$

A A A

$n \cdot A$

A A A A A A

\begin{abstract}
C A A
\end{abstract}

\begin{abstract}
A A
\end{abstract}

\begin{abstract}
A $A$
\end{abstract}
A An

A $n A$

A 


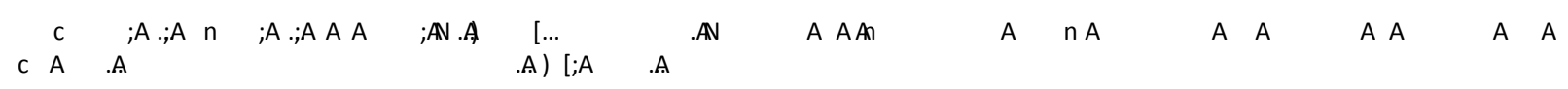
$A \stackrel{[A}{A} \begin{array}{rrr}A & A & n \\ A & A & \end{array}$
A
A A A
A $\quad$; $\quad A$
;AG ;...A A A

A

3. Approach based on the use of semantic web ontologies

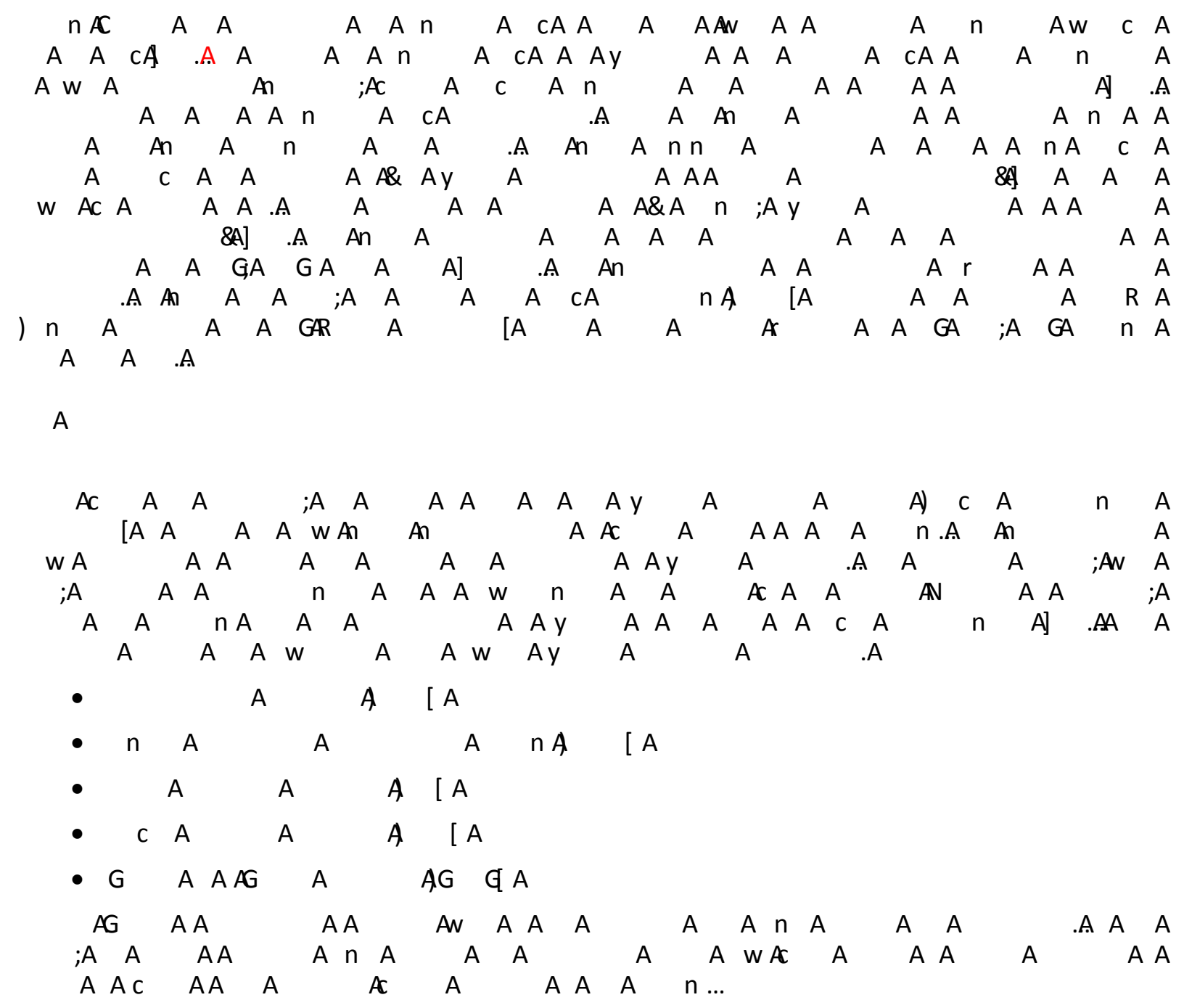

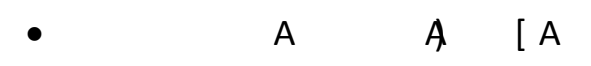

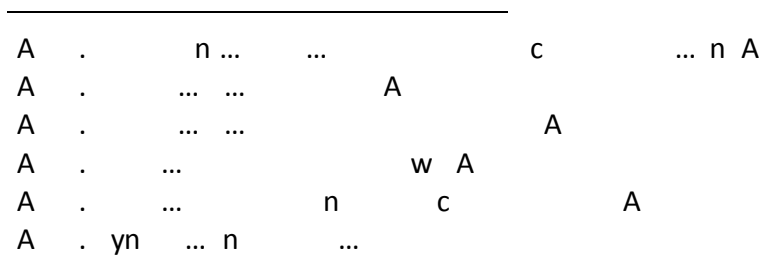




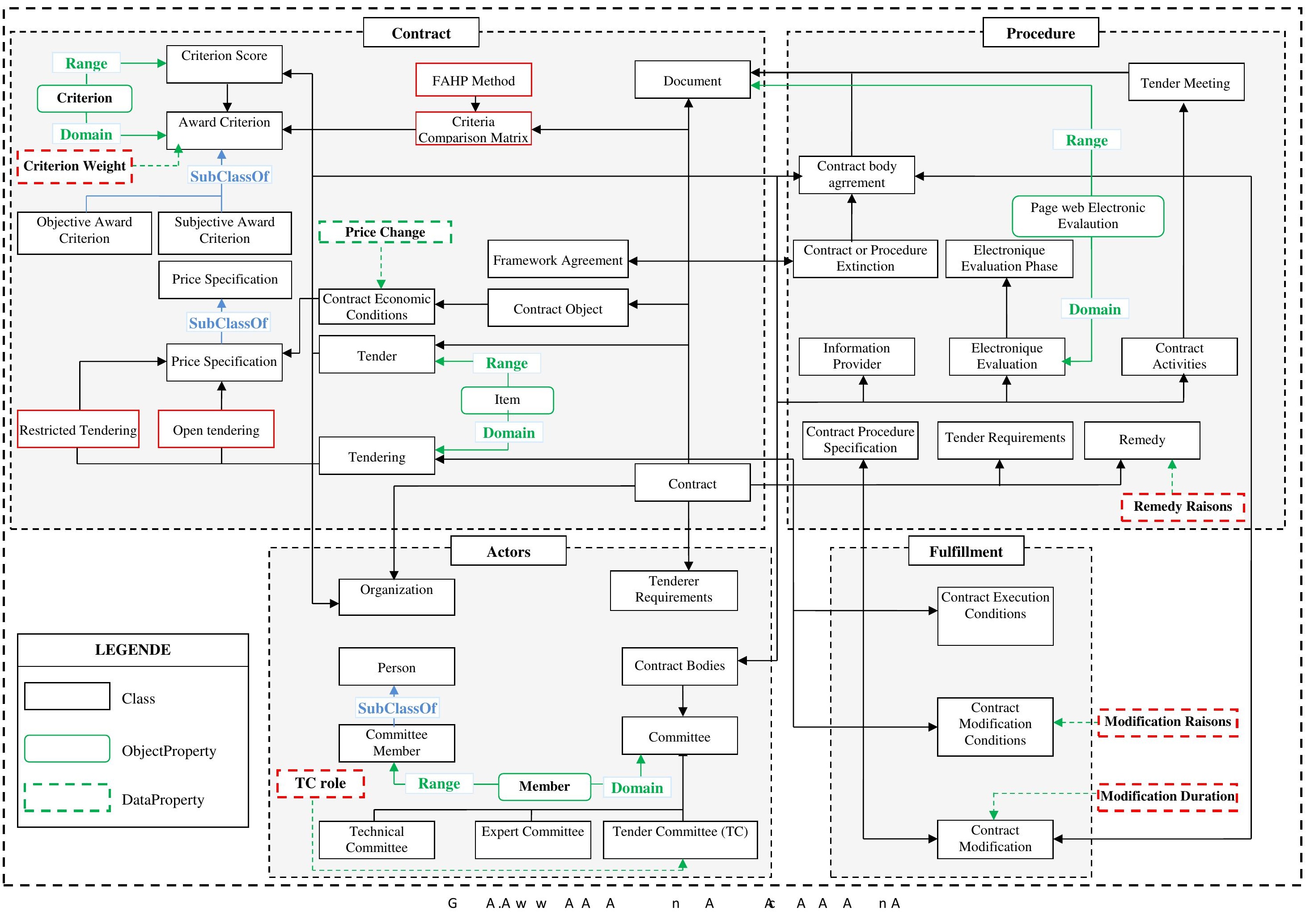


4. Multi-agents system approach

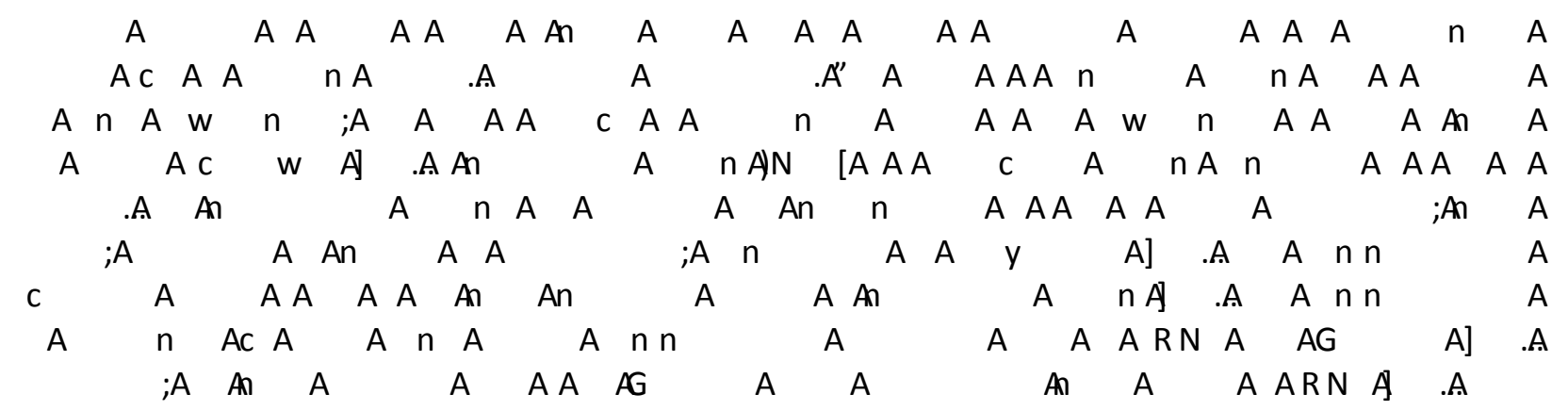

A

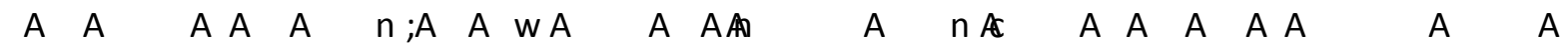

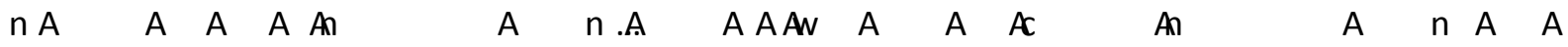

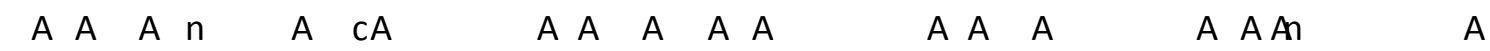
$\begin{array}{lllllllllllllllllll}n A & A & A & A & n & A & & A & A & A & A & A & A & A & A & A & A\end{array}$

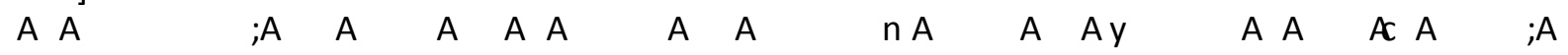
A A $\quad ; A$ A A $n n ; A A A^{\prime N} \quad$ A $n$ A A A $A$

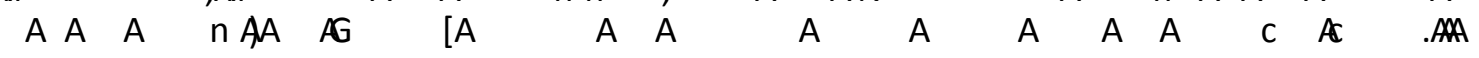

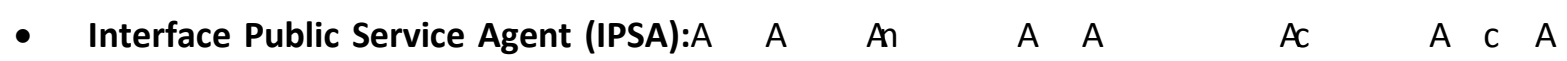
$n \quad A \quad A \quad A \quad n$.A

- Interface Enterprise Agent (IEA):A A A A A A A $\quad$ AC A $\quad$ A A A $\mathrm{A}$.A

- Interface Tender Committee Agent (ITCA):A A $\quad$ A $\quad$ A An n C A A $A$ $\begin{array}{lllllllll}n n & A & A & A & n A & A & A & A & A\end{array}$

- Planner Agent (PLA):A A A $A$ A $A \quad$ A A A $n$ A A A $\quad A \quad A$ $\begin{array}{lllllllll}A & A & A & A & \cap A & A & A & \ldots\end{array}$

- Reception Tenders Agent (RTA): A A $\quad$ C A A A A A A cn Ac A

- Selection Bidders Agent (SBA): A A A w Ar A A A $A$ A A $A$ $n \mathrm{n} \quad \mathrm{AC} \quad \mathrm{A} A \mathrm{~A} A$

A A A A A A A A

- Tenders Evaluation Agents (TEA):A An A A A A A A A A w A A .A

- Contractualization Management Agent (CMA): A A N A A r $\quad$ A A A $\quad$ A A A A A A A

- Monitoring and Control of Contract Execution Agent (MCCEA): A A A w w A A $\begin{array}{lllllllllllllll}n & A & A & A & A & A y & A A & A & A & A & A & A & A & A\end{array}$ $r \quad A \quad A A$ A C A $n$ A A A A A A

- IT Security Agent (ITSA): A A A A A A A A A $A A A$ A AAA A A A A

A A $A \quad$ A $\quad$ A .A .A 
- Users Profiles Management Agent (UPMA):A A A A A A N A w w A $\quad$;A A A A A A A

- Resources Management Agent (RMA): A A A A A A A Ac A A A $n \quad A \quad A \quad A \quad A A \quad A \quad A \quad n \ldots$

\section{Big data approach}

$\begin{array}{lllllllllllllllll}\text { A } & \text { A } & ; A & \text { A } & \text { A } & \text { A } & \text { A } & \text { A } & \text { A } & \text { A } & \text { A y } & \text { A An } & \text { A } & \text {;A }\end{array}$ $\begin{array}{llllllllllllllll}A & A & A & A & A & A & A & A & A & A & A & A & A & A & A\end{array}$ C A) Ac $\left[\begin{array}{lllllllllllllllllllll}A & A & A & A & A & A & A & A & A & A & \text { y } & A & A\end{array}\right.$ $n \quad A \quad A C A \quad A \quad A \quad A \quad A \quad A \quad A \quad A \quad n$ yA $n$ A $A$ A $\quad$ A $A$ A A $\quad A \quad A \quad$ Av $A$ A A A $; A$ C $A$ A $\quad$ A $A C A$ A $A$ A A WAn A)W $n$;AW ;AW [A] AA ;A A $A$ W $A$ $A$ A $A$ A A A $W$ A $A$ AWA $W ; A$ A A AA A A A AA C A A YA A A WA A A AWA A A A A A C A

A

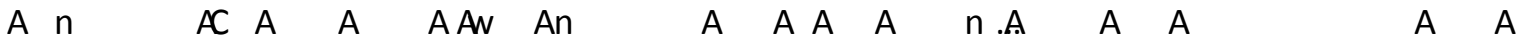
$\begin{array}{lllllllllllllll}A & A & A & \text { y A A } & \subset & A & \mathrm{~A} & \mathrm{~A} & \mathrm{~A} & \mathrm{~A} & \mathrm{~A} & \mathrm{~A} & \mathrm{~A} & \mathrm{~A} & \mathrm{~A}\end{array}$ A $A$ A A $\quad A A$ A A A A A $A$ A A A $A$ A A A A $\because A A$ A A A A A A A A A A A $n A$ A [A $A$

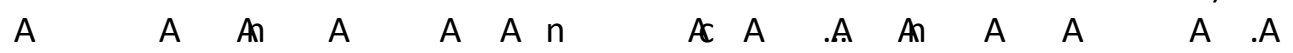

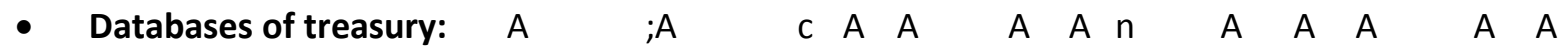
$A \quad$;A $A A$ An $A A \quad n \quad A \quad\left[\begin{array}{llllll}A & A & A\end{array}\right.$

- The Web and social networks: A A A A $\quad$ A A A cA A $A$ A $\begin{array}{lllllllllll}A A & n & A & A & A & A & A & A & A & A A & A\end{array} W A$ A A A $w \quad A A C A \quad A C A \quad A \quad A \quad n \quad A \quad n A \quad A \quad A \quad C A$

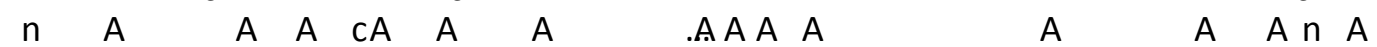
AwwA A A A $\mathrm{n}$ A A c A $A$ A $A$ A $A$ Aw A C $\quad$ A $A \begin{array}{lllllll} & \text { A } & \text { A } & \text { A } & \text { A A } & \ldots\end{array}$

- Data warehouse of price reference system: A $n$ A A A n A A $A$ $\begin{array}{lllllllllllll}A \mathrm{~A} & \text { An } & A & \mathrm{n} & \mathrm{A} & \mathrm{A} & \mathrm{A} & ; \mathrm{A} & \mathrm{A} & \mathrm{A} & \mathrm{A} w & \mathrm{~A} & \mathrm{~A}\end{array}$ A A A A A $\quad A A$ A A A $n$ A A ;A $A$ A $\begin{array}{llllllllllllllll}A & A & w & A & A & A & n & A] & A & A & A & A & A n & A & A\end{array}$

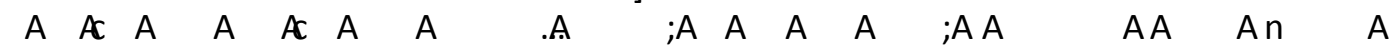

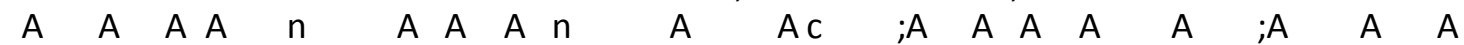
$w$ W A Aw A A A A n A Ac A A A A A $n$ A A A C "A A

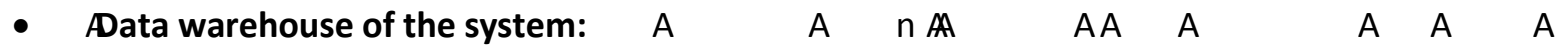

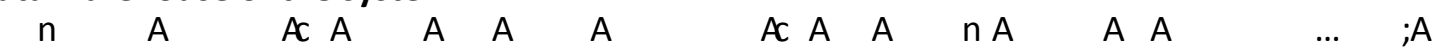
$A \quad n A$ AA AC A AA A $\cap$ A A A $n$ A A A A A A A A 


\section{Technical Architecture}

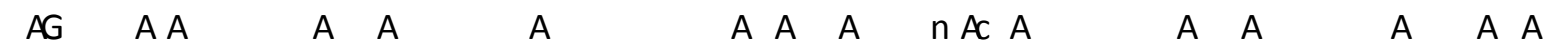
C A An $n$ A A $n$ A A A $n A$ A A $\quad A \quad$ A $n$ A A $A$ A $A A$ A A A A A A $A$ A R A A A A A A A A A A A A $\begin{array}{llllllllllllllllll}A & A & A & n & A & A & A & A & ; A A & A & A & w & n & A & A & A & A & A\end{array}$ A WAA A $\mathrm{n}$ A A A A A $\quad$;A A A A $A$ A $A$ A .A

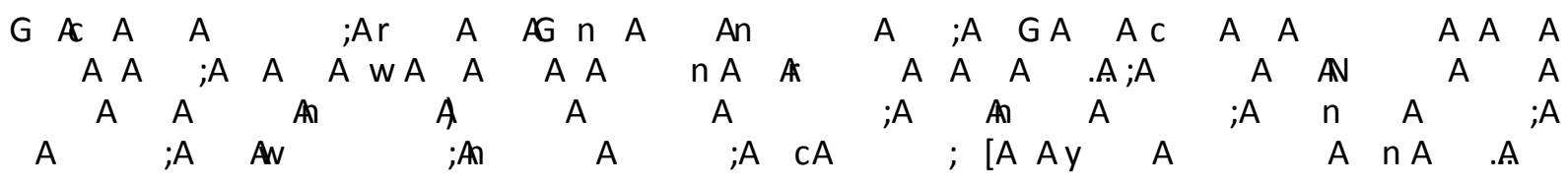
A

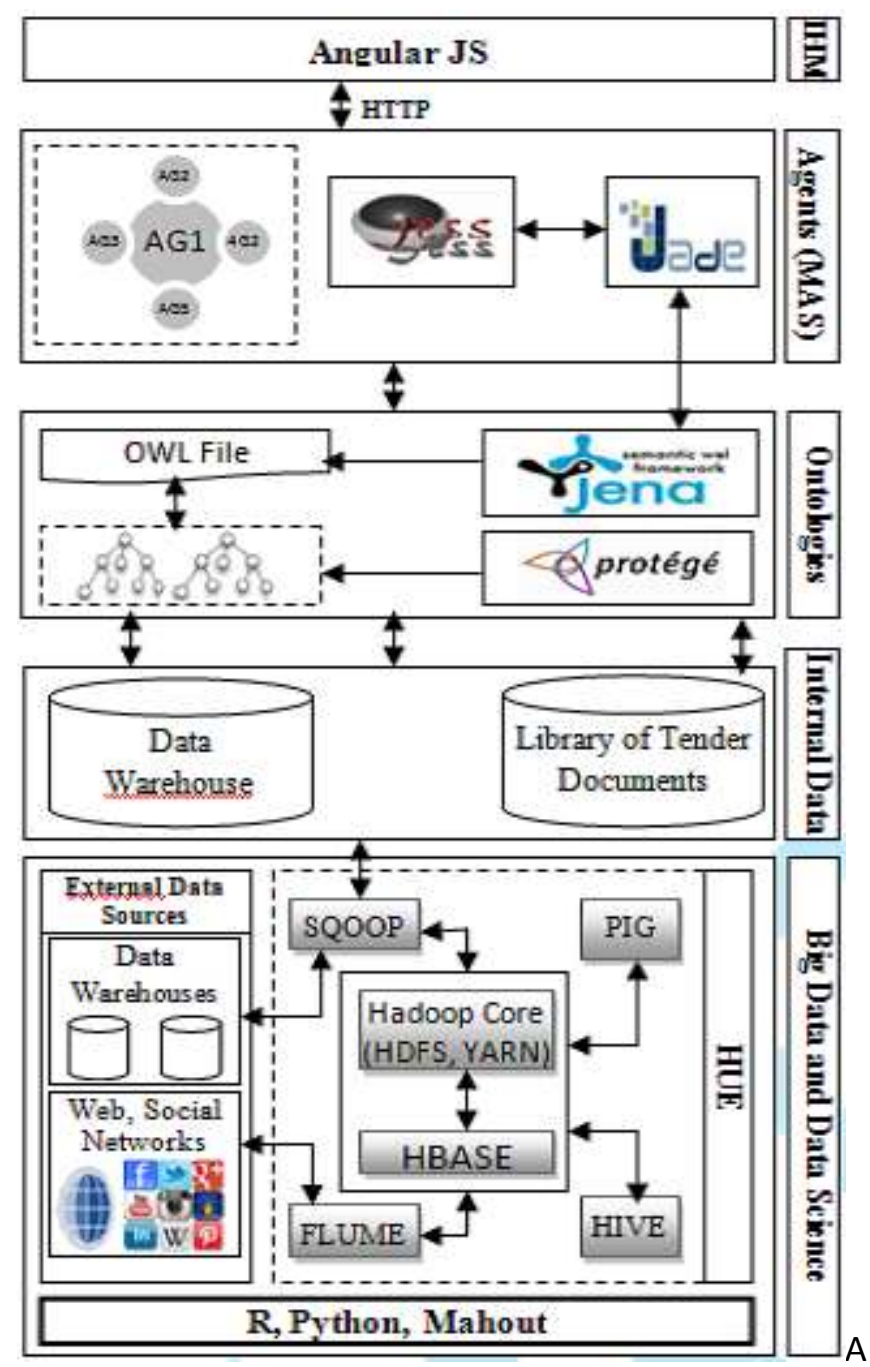

G A.A A A A A $\mathrm{A} \mathrm{A}$ 


\section{Conclusion}

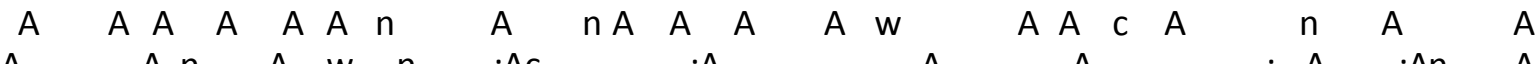

$$
\begin{aligned}
& \text { A } \quad A n \text { A } w n \text {;Ac } \quad ; A \quad \text { A } A \text {; } \quad \text { A } \quad ; A n \text { A } \\
& A \text { W A A } n \text { A A C A } n \text { A A A A A A A A } \\
& \begin{array}{llll}
\mathrm{n} & \mathrm{A} & \mathrm{n} & \mathrm{A}
\end{array}
\end{aligned}
$$

- $\begin{array}{lllllllll} & n & A & A & A & A & A & A & A\end{array}$

$\begin{array}{lllllllllllllllllll}A & A & ; A & A & A & A & A & A & A & A & A n & w A & A & n & A & A\end{array}$ $\cap A$ A A A A A A A A A A A A Al A A A Ay A $\begin{array}{llllllllllllllllll} & n A & A & A & A & A n & n & A & A & A & A & A & A & A w & A\end{array}$

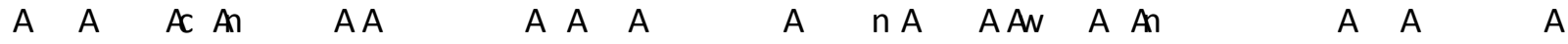
A A A $\quad$.A

A

\section{Reference}

$\begin{array}{llllllllllllllll}\mathrm{A} n & ; \mathrm{A} . ; \mathrm{A} & \mathbb{N} & ; \mathrm{A} . \mathrm{A} & {[. \mathrm{A}} & \mathrm{C} & \mathrm{A} & \mathrm{A} A & \mathrm{~A} & \mathrm{C} & \mathrm{A} & \mathrm{n} & \mathrm{A} & \mathrm{A} & \mathrm{A} & \text {.A }\end{array}$

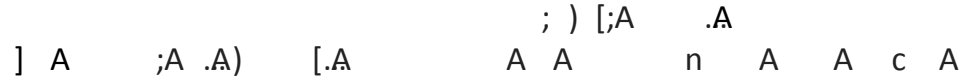

;A ) [;A .A

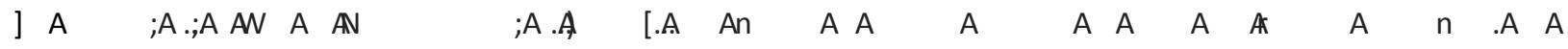

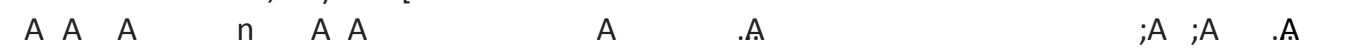

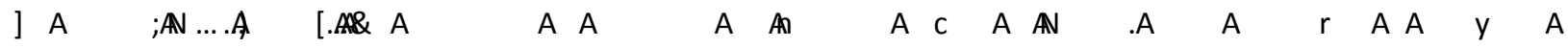
$A A \cap$ A A A A An A C A A A w A [A A A w A A A

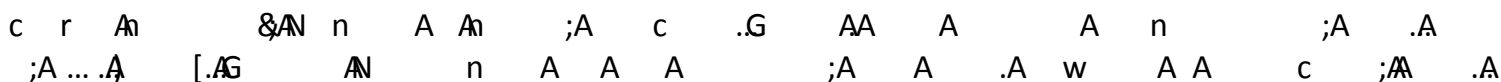

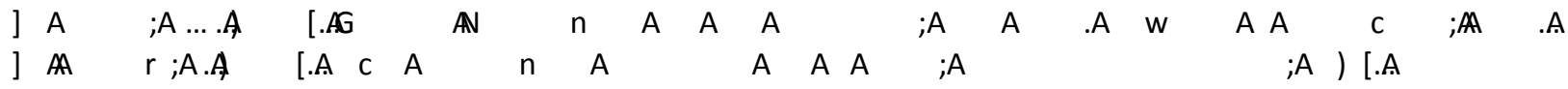

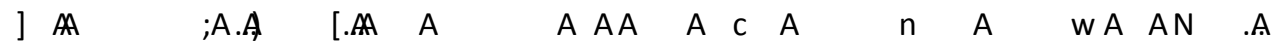

] $\begin{array}{lllllllllllllllll}A & n ; A . A & {[. A} & A & & ; A & c & A & n & ; A & A & A & n & \text {.A } & A & A w & A\end{array}$ $\begin{array}{llll}n A & A & ; A & ; A\end{array} ; \quad$ A

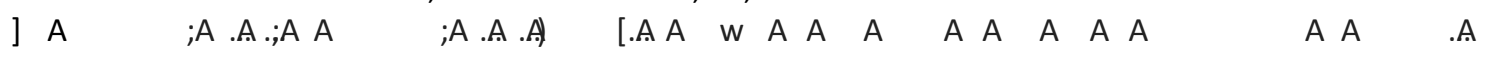

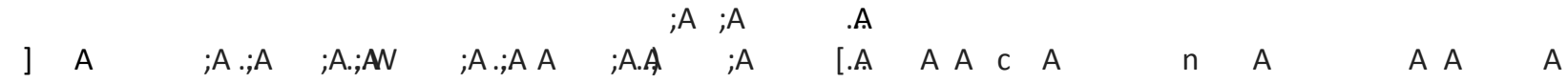

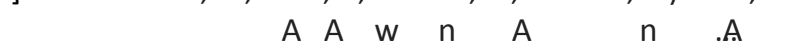

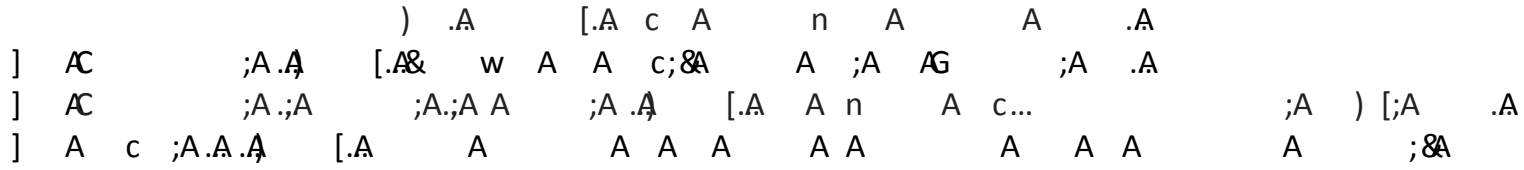
;A ) $[; A \quad$.A 


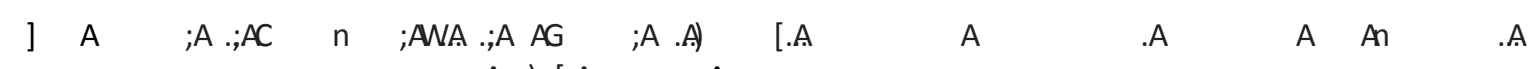
$; A)[; A \quad$ A

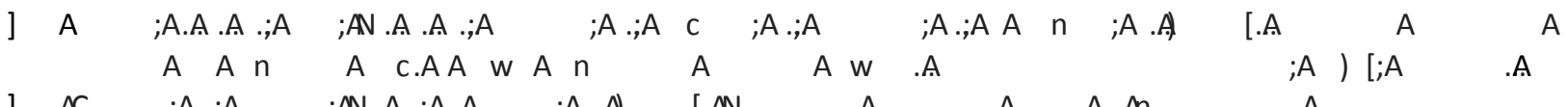
] $A C \quad ; \mathrm{A} . ; \mathrm{A} \quad ; \mathrm{AN} . \mathrm{A} . ; \mathrm{A} A \quad ; \mathrm{A} . \mathrm{A}) \quad[. \mathrm{AN} \quad \mathrm{A} \quad$ A $\quad \mathrm{A}$ An A

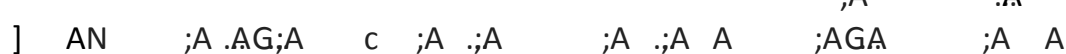

A A

A A $C$ A

] $\quad \mathbb{N} \quad A^{n \quad A} ; A$ ;A $\quad[; A \quad$ A

$\begin{array}{lll}A & A \\ A & A\end{array}$

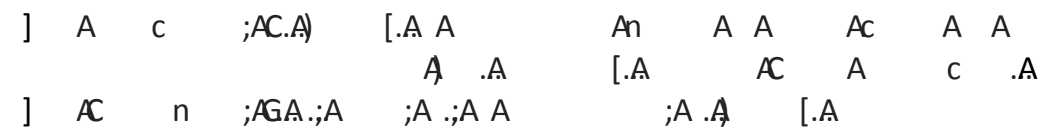

A.A $\quad$;A A W $A \quad ; A \quad$ [A A

A A

A $A$ $A A \quad$ A

] $\quad A G \quad ; A . ; \mathbb{N} \quad ; A . ; A$

] $A \quad ; A G ; A \quad C ; A ; A$ G

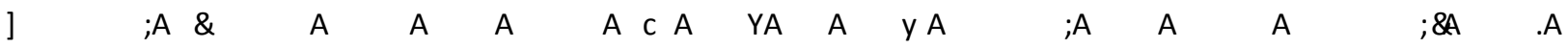
] $\mathrm{A}$ $\begin{array}{lll}\ldots \mathrm{n} & \ldots \mathrm{n} & \mathrm{C}_{\mathrm{C}}^{A}\end{array}$ AA

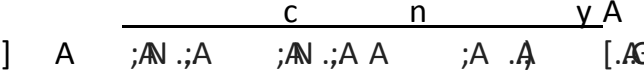
$\begin{array}{llll}A & w & A & A\end{array}$

A $A$ A $\cap A$ A $\quad C$ ;A $; A \quad$ A A

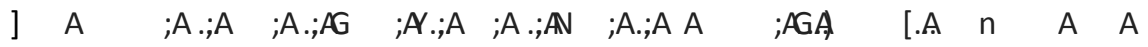
$\begin{array}{lll}A & A & \text { A }\end{array}$

] $\mathrm{A} \quad ; \mathrm{A}, ; \mathrm{A} \quad ; \mathrm{A} . ; \mathrm{A} A \mathbb{A} \quad ; \mathbb{N} . A \quad\left[\begin{array}{llll}A & A & A\end{array}\right.$ $A \quad A \quad n \quad A A$ A $\quad \begin{array}{ccc}A & \text { An } A \\ \text { A } & ; \& A\end{array}$

A $A$ A A A $\mathrm{A}$

AW .A [.A A A

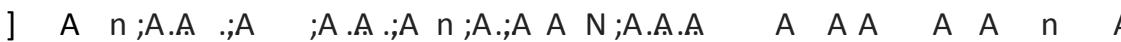
$G \quad A \quad A G n$ A A A ;A A

n

A

A 